\title{
RELATIVE BARR-RINEHART AND COTRIPLE COHOMOLOGY GROUPS ARE ISOMORPHIC
}

\author{
D. H. VAN OSDOL
}

ABstract. The theorem, stated in the title of this article, is proved.

Several people have asked about the relationship between the relative cohomology groups defined by Barr and Rinehart [3], and those of [6]. The point of this note is to show that these groups (in the cases considered by Barr and Rinehart) coincide.

In a sense, this result adds perspective on the creative tension which exists between derived functors and Ext groups. Barr has examples (see, e.g. [1]) which show that, in the theory of commutative rings, the functor which classifies extensions is definitely not a derived functor. By the results of Beck [4] and Duskin [5] (see also [6]) any algebraic theory defines a cohomology theory, the "cotriple-derived functors", which classifies extensions. The paper of Barr and Rinehart can thus be viewed as a statement that for certain theories these intrinsically defined groups can equally well be computed by the more classical approach of "ordinary" derived functors on a module category. This note shows that the Barr-Rinehart relative groups $\operatorname{Ext}_{R}^{\prime \prime}\left(S / S^{2}, M\right)$ also appear naturally in the cotriple theory. This raises the following unanswered question: what conditions on an algebraic theory make it possible to compute the intrinsic long exact cohomology sequences via ordinary derived functors? Here we shall give conditions, but they are not really conditions on the theory.

Let us quickly reassemble the relevant data from [3 and 6] and set forth our assumptions. Let $U: \underline{C} \rightarrow \underline{B}, F: \underline{B} \rightarrow \underline{C}$ be a tripleable adjoint pair and $(G=F U, \varepsilon, \delta)$ the corresponding cotriple on $\underline{C}$. Let $H^{*}(-, M)$ be the $G$-derived functors, with $M$ an abelian group in $\underline{C}$. Suppose $\underline{B}$ (and hence $\underline{C}$ ) has finite inverse limits, and let $\phi$ : $T \rightarrow R$ be a $U$-split epimorphism in $\underline{C}$. Let $k^{0}, k^{1}: P \rightarrow T$ be a kernel pair of $\phi$ and $k^{0}, k^{1}, k^{2}: K \rightarrow P$ be a kernel triple of $\left(k^{0}, k^{1}\right)$. Let

$$
\operatorname{Hom}_{n}\left(k^{0}, k^{1} ; M\right)=\left\{f: G^{n+1} P \rightarrow M \mid f \circ G^{n+1} k^{0}+f \circ G^{n+1} k^{2}=f \circ G^{n+1} k^{1}\right\}
$$

for $n \geqslant-1$, and let $\mathrm{Ab}(\underline{X})$ denote the category of abelian group objects in category $\underline{X}$. Then $\operatorname{Hom}_{n}\left(k^{0}, k^{1} ;-\right): \mathrm{Ab}(\underline{C}) \rightarrow \mathrm{Ab}(\underline{\text { Sets }})$ is a functor; suppose that

$$
\operatorname{Hom}_{-1}\left(k^{0}, k^{1} ;-\right) \text { is representable by } S / S^{2} \text { in } \mathrm{Ab}(\underline{C}) \text {. }
$$

Received by the editors December 20, 1982 and, in revised form. May 17, 1983.

1980 Mathematics Subject Classification. Primary 18G50: Secondary 20J05, 13D03. 16A61. 
Caution: $S / S^{2}$ is merely notation for the representing object and will not in general "be" $S$ modulo its square; the notation is that of Barr and Rinehar. The groups $\operatorname{Hom}_{n}\left(k^{0}, k^{1} ; M\right)$ define a subcomplex of $\underline{C}\left(G^{*+1} P, M\right)$ and we denote its $m$ th homology group by $H^{m}(\phi ; M)$.

Let 2 be the class of all monomorphisms in $\mathrm{Ab}(\underline{C})$ which are kernels of $U$-split epimorphisms in $\mathrm{Ab}(\underline{C})$; here $U$ is also being used to denote the functor $\operatorname{Ab}(\underline{C}) \rightarrow$ $\mathrm{Ab}(\underline{B})$ induced by $U: \underline{C} \rightarrow \underline{B}$. Note that Barr and Rinehart denote by 2 the class of all monomorphisms in $\mathrm{Ab}(\underline{C})$ which are $U$-split in $\mathrm{Ab}(\underline{B})$, but in their examples the two definitions yield the same class. An object $M$ in $\mathrm{Ab}(\underline{C})$ is 2 -injective if $\operatorname{Ab}(\underline{C})\left(N^{\prime}, M\right) \rightarrow \operatorname{Ab}(\underline{C})(N, M)$ is surjective whenever $N \rightarrow N^{\prime}$ is in 2 . We suppose

$$
\operatorname{Ab}(\underline{C}) \text { has enough } 2 \text {-injectives, and }
$$

With the technicalities now behind us, we proceed to the main result. The following consequence of our definition of 2 is vital.

Lemma. $U: \mathrm{Ab}(\underline{C}) \rightarrow \mathrm{Ab}(\underline{B})$ preserves quotients of members of 2 ; in fact they are split in $\mathrm{Ab}(\underline{B})$.

Proof. Let $f: A^{\prime} \rightarrow A$ be in 2 , and $p: A \rightarrow C$ the $U$-split epimorphism in $\operatorname{Ab}(\underline{C})$ of which it is the kernel. Then any quotient of $f$ in $\mathrm{Ab}(\underline{C})$ is uniquely isomorphic to $p$, so we need that $U p$ is a quotient of $U f$ in $\operatorname{Ab}(\underline{B})$. But $U A^{\prime} \underset{U O}{\stackrel{U f}{\Rightarrow}} U A \stackrel{U p}{\rightarrow} U C$ is a split coequalizer and hence we are finished. Q.E.D.

THEOREM. For $n \geqslant 0, H^{n}(\phi ;-)$ and $\operatorname{Ext}_{R}^{n}\left(S / S^{2},-\right)$ are isomorphic functors $\mathrm{Ab}(\underline{C}) \rightarrow \mathrm{Ab}($ Sets $)$; here $\mathrm{Ext}^{n}$ is the nth derived functor of $\mathrm{Ab}(\underline{C})\left(S / S^{2},-\right)$ using the injective class $\overline{2}$ as in [3].

Proof. It is only necessary (thanks to assumption (2) above) to prove that $H^{*}(\phi ;-)$ is a 2 -exact cohomological $\delta$-functor augmented over $\operatorname{Ab}(\underline{C})\left(S / S^{2},-\right)$. By assumption (1), $\mathrm{Hom}_{-1}\left(k^{0}, k^{1} ;-\right) \simeq \mathrm{Ab}(\underline{C})\left(S / S^{2},-\right)$; but by 3.5 of [6], $0 \rightarrow$ $\operatorname{Hom}_{-1}\left(k^{0}, k^{1} ;-\right) \rightarrow \operatorname{Hom}_{0}\left(k^{0}, k^{1} ;-\right) \rightarrow \operatorname{Hom}_{1}\left(k^{0}, k^{1} ;-\right)$ is exact, so $\operatorname{Hom}_{1}\left(k^{0}, k^{1} ;-\right) \simeq H^{0}(\phi ;-)$ and hence $\operatorname{Ab}(\underline{C})\left(S / S^{2},-\right) \simeq H^{0}(\phi ;-)$. Next suppose $M$ is 2 -injective and $n \geqslant 1$; then by 3.7 of $[6], H^{n}(T, M) \rightarrow H^{n}(\phi ; M) \rightarrow$ $H^{n+1}(R, M)$ is exact. Because of assumption $(3), H^{n}(T, M)=H^{n+1}(R, M)=0$ and thus $H^{n}(\phi ; M)=0$. Finally, let $0 \rightarrow M^{\prime} \stackrel{f}{\rightarrow} M \rightarrow M^{\prime \prime} \rightarrow 0$ be a 2 -exact sequence in $\mathrm{Ab}(\underline{C})$; by the lemma, if $n \geqslant 0$ and $X$ is in $\underline{C}$ then $0 \rightarrow \underline{C}\left(G^{n+1} X, M^{\prime}\right) \rightarrow$ $\underline{C}\left(G^{n+1} X, M\right) \rightarrow \underline{C}\left(G^{n+1} X, M^{\prime \prime}\right) \rightarrow 0$ is split exact in $\mathrm{Ab}($ Sets $)$, since it is naturally isomorphic to $0 \rightarrow \underline{B}\left(U G^{n} X, U M^{\prime}\right) \rightarrow \underline{B}\left(U G^{n} X, U M\right) \rightarrow \underline{B}\left(\overline{U G}^{n} X, U M^{\prime \prime}\right) \rightarrow 0$. Since $\phi: T \rightarrow R$ is a $U$-split epimorphism, $\underline{C}\left(G^{n+1} R, N\right) \rightarrow \underline{C}\left(G^{n+1} T, N\right)$ is a split monomorphism in $\mathrm{Ab}($ Sets) for any $N$ in $\mathrm{Ab}(\underline{C})$; recall that its quotient is $\operatorname{Hom}_{n}\left(k^{0}, k^{1} ; N\right)$. $\overline{\mathrm{A}}$ simple nine-lemma type argument now shows that $0 \rightarrow$ $\operatorname{Hom}_{n}\left(k^{0}, k^{1}, M^{\prime}\right) \rightarrow \operatorname{Hom}_{n}\left(k^{0}, k^{1} ; M\right) \rightarrow \operatorname{Hom}_{n}\left(k^{0}, k^{1} ; M^{\prime \prime}\right) \rightarrow 0$ is exact for $n \geqslant$ 0 . From the resulting short exact sequence of cochain complexes we get a long exact sequence $\cdots \rightarrow H^{n}(\phi ; M) \rightarrow H^{n}\left(\phi ; M^{\prime \prime}\right) \rightarrow H^{n+1}\left(\phi ; M^{\prime}\right) \rightarrow H^{n+1}(\phi ; M) \rightarrow \cdots$, and the connecting homomorphisms are natural. Q.E.D. 
It remains to show how to use this theorem to verify that the relative cotriple groups $H^{n}(\phi ; M)$ are isomorphic to the Barr-Rinehart groups $\operatorname{Ext}_{R}^{n}\left(S / S^{2}, M\right)$. Let $C_{\text {and }} \bigodot_{0}$ be as in [3], with $U^{\prime}: \bigodot \rightarrow \bigodot_{0}$ the forgetful functor and $F^{\prime}: \bigodot_{0} \rightarrow \bigodot$ its left adjoint (as described in [3]). For $R$ in $\bigodot$ let $\underline{C}=\bigodot / R, \underline{B}=\bigodot_{0} / U^{\prime} R$ and $U: \underline{C} \rightarrow \underline{B}$, $F: \underline{B} \rightarrow \underline{C}$ the extensions of $U^{\prime}, F$ to these comma categories (e.g. $F\left(S \rightarrow U^{\prime} R\right)=F^{\prime} S$ $\rightarrow F^{\prime} U^{\prime} R \stackrel{\varepsilon^{\prime} R}{\rightarrow} R$ ). It is by now well known that $U^{\prime}$ and hence $U$ are tripleable in each of the cases $A, S, L$, and $G$ of [3] (which are, respectively, unitary $K$-algebras, supplemented unitary $K$-algebras, Lie algebras over $K$, and groups), and that the $H^{n}(X, M)$ defined by Barr and Rinehart agree with the resulting cotriple cohomology groups (see e.g. [2]). Thus assumption (3) above is true. Much as in $§ 7$ of [6], one can show that $S / S^{2}$ as defined by Barr and Rinehart represents $\operatorname{Hom}_{-1}\left(k^{0}, k^{1} ;-\right)$ in cases $A, S, L$, and $G$. This is assumption (1) above. Finally, assumption (2) is proved in [3].

\section{REFERENCES}

1. M. Barr, A note on commutative algebra cohomology, Bull Amer. Math. Soc. 74 (1968), 310-313.

2. M. Barr and J. Beck, Homology and standard constructions, Seminar on Triples and Categorical Homology Theory, Springer, Berlin, 1969, pp. 245-335.

3. M. Barr and G. Rinehart, Cohomology as the derived functor of derivations, Trans. Amer. Math. Soc. 122 (1966), 416-426.

4. J. Beck, Triples, algebras, and cohomology, Ph. D. dissertation, Columbia University, 1967.

5. J. Duskin, Simplicial methods and the interpretation of "triple" cohomology, Mem. Amer. Math. Soc. No. 3 (1975).

6. D. Van Osdol, Long exact sequences in the first variable for algebraic cohomology theories, J. Pure Appl. Algebra 23 (1982), 271-309.

Department of Mathematics, University of New Hampshire, Durham, New Hampshire 03824 